\section{Balkankonflikt, Immigration und Kreuzschmerz}

Die in dieser Ausgabe zusammengestellten Artikel zum Thema "Immigrationskreuzschmerz im aktuellen Kontext mit dem Balkankonflikt" sind aufgrund einer Fortbildungstagung in Valens* entstanden.

Otto Knüsel, Organisator der Tagung, umreisst die Thematik: Die Prävalenz der Immigrationskreuzschmerzen nimmt tendenziell zu. Das grosse Problem ist die Chronifizierung der Schmerzen und die damit verbundene zunehmende Invalidisierung, womit einmal mehr die Grenzen unseres Gesundheitssystems sichtbar werden. Bei den Patienten aus Ex-Jugoslawien kommt der Sozialintegration und dem dabei notwendigen Verständnis der politischhistorischen Hintergründe eine besondere Bedeutung zu.

Cyrill Stieger, Auslandredaktor der NZZ, skizziert den Zerfall des Vielvölkerstaates nach dem Tod Titos, mit den Folgen bis in die jüngste Gegenwart. Die Situation im Kosovo und in Bosnien werden speziell beleuchtet: ethnische Ausgrenzungen, Krieg, das heutige westliche Protektorat und die ungewisse Zukunft.

Auf dem Hintergrund dieser historischen Entwicklung beschreibt Nada Boškovska, Slawistin und Historikerin, die Immigration der Menschen aus Jugoslawien in die Schweiz. Von 1980 bis 1990 hat sich die Zahl der in der Schweiz lebenden Jugoslawen verzehnfacht, heute leben etwa 300000 Ex-Jugoslawen in der Schweiz, dabei ist der Anteil an Albanern stetig gestiegen und beträgt heute etwa 2/3 dieser Bevölkerungsgruppe. Sie prägen das Bild des "Jugoslawen" in der Schweiz. Die Beschreibung der ursprünglichen Lebensweisen und Wertvorstellungen hilft zu verstehen, warum gerade diese Menschen Integrationsprobleme haben, warum ihre traditionellen Verhaltensmuster in der Schweiz versagen, warum daraus Orientierungslosigkeit und zutiefst angegriffenes Selbstwertgefühl folgen.

Matthias Reiber, Psychosomatiker, verdeutlicht am Beispiel des Schmerzes, dass Natur und Kultur miteinander verwoben sind. Der Begriff Schmerz erfährt einen entsprechenden Wandel im Verlauf der Geschichte. Unter diesem Aspekt bekommt Schmerz als Kommunikationsmittel eine besondere Bedeutung: Schmerz will verstanden werden, und zum Verstehen ist der Einbezug des Menschen in seiner gesamten Situation notwendig: siehe Immigrationskreuzschmerz. Womit wir wieder beim Thema wären.

In diesem Zusammenhang haben die Kollegen Jan Kool, Peter Oesch und Stefan Bachmann die Frage gestellt: Ist die Nationalität prädiktiv für die Beurteilung der körperlichen Leistungsfähigkeit und für das Rehabilitationsresultat? Ihre Antwort liegt nun in Form von drei Originalarbeiten vor. Die Redaktion hat sich entschlossen, diese zu veröffentlichen, obwohl sie, genau betrachtet, gewisse methodologische Mängel aufweisen, welche eine Veröffentlichung in Frage hätten stellen können; es sei nun dem Leser überlassen, die Schlussfolgerungen selbst kritisch zu prü- fen. Der besondere Wert dieser Arbeiten scheint mir aber darin zu liegen, dass hier erstmals der Versuch unternommen wird, Zahlen und Fakten zu dieser sensiblen Thematik zu erarbeiten. Damit wird zumindest der Weg aufgezeigt, auf welchem weitere, präzisere und aussagekräftigere Untersuchungen folgen könnten. Oder besser: folgen sollten. Denn, was kann Medizin besseres bieten, als verbreiteten und dazu noch meinungsbildenden Stammtischparolen das entgegenzusetzen, was sie immer noch auszeichnet: die Denk- und Handelsweise einer empirischen Wissenschaft. Wenn diese Arbeiten weitere Untersuchungen anregen würden, hätten sie in meinen Augen ihren wichtigsten Zweck erfüllt.

Regula Weiss, Psychiaterin, erörtert anhand der Geschichte einer psychoanalytisch orientierten Psychotherapie Themen und Konflikte, welche im Migrationsprozess gehäuft auftreten. Sie plädiert für den systematischen Einbezug migrationsspezifischer lebensgeschichtlicher Aspekte.

Heinz Herzer, Arzt IV-Stelle St. Gallen, untersucht die Entwicklung der Rentenzusprachen im Kanton St. Gallen in den Jahren 1994 und 1999. Bis zum Jahre 1997 findet eine Verdoppelung der Rentengesuche und Zusprachen statt, seit 1998 ist diese Entwicklung etwas rückläufig. Der Ausländeranteil der Neurentner steigt in der untersuchten Zeitspanne von $25 \%$ auf $34 \%$ an. Letztlich muss aber konstatiert werden, dass die zunehmende Invalidisierung der Gesellschaft vorläufig ungeklärt bleibt und dass eine Reduktion der Problematik auf eine Ausländerfrage weder medizinisch noch ethisch vertretbar ist.

Thomas Wieland, Leiter Abteilung Migration, Amt für Soziales in St. Gallen, bespricht die Belastung des Asylund des Gesundheitswesens durch Asylsuchende bzw. Kriegsvertriebene aus Jugoslawien und betont gleichzeitig, dass ein menschenwürdiger Umgang mit allen Bewohnerinnen und Bewohnern unseres Landes und die Einhaltung der Menschenrechte oberste Maxime bleiben muss.

Der Artikel von Alessandro Huber, Psychiater, stammt nicht aus der Fortbildungstagung in Valens, wird aber hier ebenfalls veröffentlicht, weil er thematisch das Bild ergänzt. Seine Bitte: Bitte denken Sie nicht sofort, dass schon die Tatsache der Zuwanderung die ganze Symptomatik erklären kann.

Wir hoffen, dass diese Beiträge dazu dienen, betroffene Patientinnen und Patienten besser zu verstehen und dass der medizinische Standpunkt in der politischen Diskussion vermehrt berücksichtigt werde.

Markus Trutmann Chefredaktor

\footnotetext{
* 2. Valenser Frühlingssymposium «Der Immigrationskreuzschmerz im aktuellen Kontext mit dem Balkankonflikt». Rheuma- und Rehabilitationsklinik Valens, 23. März 2000.

Die Veranstaltung wurde von der Firma Knoll AG, 4410 Liestal, unterstützt. Dies ist nicht selbstverständlich, denn es geht hier für einmal nicht um den üblichen produktebezogenen Zirkus, unmittelbarer Gewinn schaut dabei nicht heraus. Die Firma Knoll verdient Anerkennung.
} 